УДК $532+541.11$

\title{
РАСЧЕТНАЯ ОЦЕНКА ТЕПЛОФИЗИЧЕСКИХ СВОЙСТВ АЗОТА, КАК РАБОЧЕГО ТЕЛА ПОРШНЕВОГО КРИОДВИГАТЕЛЯ. ЧАСТЬ III. ВЫЧИСЛЕНИЕ ЭНТАЛЬПИИ И ЭНТРОПИИ
}

\section{Левтеров А.М., Умеренкова К.Р.}

Институт проблем машиностроения им. А.Н. Подгорного НАН Украины, ул. Пожарского, 2/10, 2. Харьков, 61046

У статті викладено оригінальний метод та результати розрахунку теплофізичних властивостей азоту, що використовується як робоче тіло для транспортних поршневих двигунів.
В статье приведен оригинальный метод и результаты расчета теплофизических свойств азота, используемого в качестве рабочего тела для транспортных поршневых установок.
In article the original method and results of calculation thermo physical properties of the nitrogen used as a working body for transport piston installations is resulted.

Библ. 10, табл. 2, рис. 2.

Ключевые слова: поршневой криодвигатель, азот, теплофизические характеристики, рабочее тело, энтальпия, энтропия, математическая модель.

$D$ - массовая плотность;

$\widetilde{D}$ - параметр приведения для массовой плотности;

$E$ - параметр приведения для температуры;

$F, f$ - свободная энергия;

$h$-удельная энтропия;

$I$ - групповой интеграл;

$k$ - постоянная Больцмана;

$M$ - молярная масса;

$N$ - число частиц;

$N_{\text {А }}$ - число Авогадро;

$p$ - давление;

$\widetilde{P}$ - параметр приведения для давления;

$R=R_{0} / M$ - газовая постоянная;

$R_{0}$ - универсальная газовая постоянная;

$u$ - потенциал взаимодействия;

$T$ - температура;

$V$ - объем;

$z$ - фактор сжимаемости;

$\beta=1 /(k T)$;

Состояние проблемы. Пневматические силовые установки для автомобилей, в которых используется газ высокого давления, стали объектом интенсивных исследований в последние несколько лет и рассматриваются как один из перспективных вариантов для автомобилей малой и средней грузоподъемности. Сюда относятся и криогенные силовые установки, в которых $\varepsilon, \sigma-$ параметры потенциала межмолекулярного взаимодействия;

$\mu$ - химический потенциал;

$\eta$ - параметр упаковки;

$\rho=N / V-$ плотность числа частиц.

Индексы верхние:

* - приведенные величины.

\section{Индексы нижние:}

0 - нулевое приближение;

1 - первый порядок теории возмущений;

2 - второй порядок теории возмущений;

$s t$ - стандартное;

$L-$ жидкость;

$S$ - насыщенный пар;

$V$ - пар;

ДВС - двигатель внутреннего сгорания;

РТ - рабочее тело;

ХНАДУ - Харьковский национальный автомобильно-дорожный университет.

используются низкотемпературные пневматические двигатели, а рабочим телом для них является жидкий азот или воздух, хранящийся в криостатах на борту автомобиля и подогреваемый до температуры окружающей среды в теплообменниках [1-3]. Отмечено, что сжиженный газ обеспечивает большую работу на единицу массы рабочего тела по сравнению с аналогичным по- 
казателем сжатого газа.

Жидкий азот получают на воздухоразделительных установках из атмосферного воздуха, где его запасы практически не ограничены. Кроме того, азот является экологически безопасным и химически инертным газом, что позволяет использовать криогенные силовые установки даже в закрытых помещениях повышенной пожароопасности, в том числе при работе с огнеопасными веществами.

Первые экспериментальные автомобили с криодвигателями, использующимися в авиационной промышленности уже созданы в США и по мнению специалистов имеют прекрасную перспективу в будущем. Представляют также интерес разработки малолитражных автомобилей французской компании Motor Development International. В Украине инженерно-конструкторские работы по разработке высокоэффективного автомобильного пневмодвигателя для криогенной силовой установки ведутся в ХНАДУ [3].

К преимуществам использования криогенных силовых установок в автомобилях относятся:

- пожаробезопасность;

- экологическая чистота;

- возможность рекуперации энергии;

- доступная технология производства;

- доступность и относительно низкая стоимость рабочего тела (сжиженный или сжатый азот, воздух);

- экономическая целесообразность (стоимость 1 км пробега меньше, чем для ДВС) [3, 4];

- возможность массового применения в городах

с высокой плотностью населения и автотранспорта [5].

В настоящее время предполагается разрабатывать автомобили с пневматической (криогенной) силовой установкой для использования в густонаселенных районах городов в качестве маршрутных такси, небольших грузовых автомобилей, машин-холодильников, а также специальных машин для обслуживания аэропортов [6], шахт и взрывоопасных производств.

Анализ исследований и публикаций. Проблема создания эффективных методов расчета термодинамических свойств веществ, которые одновременно применимы для газообразного и жидкого состояний, до последнего времени ос- тавалась нерешенной.

Существовали различного рода прогностические алгоритмы или упрощенные модельные методы, обзор которых приведен в работе [7]. Данные методы расчетов обеспечивали приемлемые результаты для отдельных термодинамических характеристик в ограниченных областях состояний, однако в целом оказывались неудовлетворительными, поскольку для набора свойств погрешности их описания существенно превышали экспериментальные ошибки.

Постановка задачи. Такое состояние проблемы обусловило необходимость развития статистико-механических методов описания свойств молекулярных веществ, в рамках которых используется минимум исходных данных и параметров. Одним из важных условий успешного использования азота в качестве РТ является создание современных инженерных методов расчета его термодинамических свойств. Эти методы расчета должны удовлетворять одновременно таким требованиям, как точность и возможность применения в широком диапазоне давлений и температур, а также доступность использования результатов в режиме текущего времени, т.е. в процессе управления режимами работы двигателя. Проводимые исследования посвящены применению оригинальной модифицированной схемы термодинамической теории возмущений [7] для описания энтальпии и энтропии азота.

\section{Метод и результаты расчета.}

Свободная энергия $F=N \cdot f$ системы $N$ частиц, взаимодействующих посредством исходного потенциала $u(r)$, при $T$ и $\rho$ имеет вид

$\beta f=\beta f_{0}+\rho^{*}\left(I_{1}+I_{2} / T^{*}\right) / T^{*}+\ldots$,

где $\rho^{*}=\rho \sigma^{3}$. Величина $f_{0}$ представляет собой удельную (на одну частицу) свободную энергию системы ТС. $I_{1}$ и $I_{2}$ - групповые интегралы первого и второго порядков теории возмущений [8].

Термодинамические свойства азота получены на базе рассчитанных согласно (1) поверхностей свободной энергии $f(T, V)$ или $F(T, \rho)$ с использованием стандартных термодинамических соотношений. Начальным этапом расчетов является определение плотности. Плотности жидкой $D_{L}$ и паровой $D_{V}$ фаз вещества на линии 
насыщения определяются из условий равенства давлений и химических потенциалов фаз:

$$
\left\{\begin{array}{l}
p\left(D_{L}, T\right)-p\left(D_{V}, T\right)=0 ; \\
\mu\left(D_{L}, T\right)-\mu\left(D_{V}, T\right)=0
\end{array}\right.
$$

а плотность вещества $D(p, T)\left[\kappa / / \mathrm{M}^{3}\right]$ в однофазной области в термодинамическом состоянии $p[\mathrm{MПа]} \mathrm{и} T[\mathrm{~K}]-$ из уравнения:

$p(D, T)-p=0$.

Приведем выражения для основных свойств. Давление $p=\widetilde{P} T^{*} \rho^{*} z$.

Фактор сжимаемости $z=10^{3} p / R T D$

$z=1+2 \eta \frac{2-\eta}{(1-\eta)^{3}}+\chi_{1}[i+1]$

Свободная энергия $F=R T(\beta f)$

$\beta f=\beta h_{0}-s_{0}-1-\ln z_{N}+\eta \frac{4-3 \eta}{(1-\eta)^{2}}+\chi_{1}[1]+\chi_{2}[1]$.

Внутренняя энергия $U=R T(\beta u)$

$\beta u=\beta h_{0}-1+\chi_{1}[k+1]+\chi_{2}[j+1]$.

Энтропия $S=R s(s=\beta u-\beta f)$

$s=s_{0}+\ln z_{N}-\eta \frac{4-3 \eta}{(1-\eta)^{2}}+\chi_{1}[k]+\chi_{2}[j]$.

Энтальпия $H=R T(\beta h)(\beta h=\beta u+z)$

$\beta h=\beta h_{0}+2 \eta \frac{2-\eta}{(1-\eta)^{3}}+\chi_{1}[i+k+2]+\chi_{2}[j+1]$.

Энергия Гиббса $G=R T(\beta \mu)(\mu=f+z / \beta)$

$\beta \mu=\beta h_{0}-s_{0}-\ln z_{N}+\eta \frac{8-9 \eta+3 \eta^{2}}{(1-\eta)^{3}}+\chi_{1}[i+2]+$

$+\chi_{2}[1]$

Здесь $\eta=0,4177 \rho^{*} ; \rho^{*}=D / \widetilde{D}, T^{*}=T / E, p^{*}=p / \widetilde{P}$, $\widetilde{D}=M /\left(N_{A} \sigma^{3}\right), E=\varepsilon / k, \widetilde{P}=R E \widetilde{D}$. Величины выражены в следующих единицах: $R-$ кДж/(кг $\cdot \mathrm{K})$;

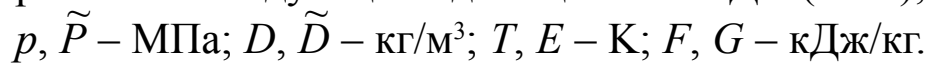

Для азота: $E=97,55 \mathrm{~K}, \sigma=3,5996 \cdot 10^{-10} \mathrm{м}[7], \widetilde{D}=$ $=997,38 \kappa г / \mathrm{M}^{3}, \widetilde{P}=8,877 \mathrm{MПа,} R=0,2968$ кДж/ (кг $\cdot \mathrm{K})$.

Формы $\chi_{1}[n]$ и $\chi_{2}[n]$ определяются согласно выражениям, приведенным в работе [7].

В выражениях (1) - (9) $z_{N}=10^{3} p_{s} / R T D-$ функция нормировки на стандартное давление $p_{s t}=$ $=0,101325$ МПа, а величины $h_{0}, c_{p 0}, s_{0}$ - удельные (приходящиеся на одну молекулу) энтальпия, энтропия и изобарная теплоемкость в идеальногазовом состоянии.

Для определения $h_{0}, c_{p 0}, s_{0}$ использованы выражения Пассата-Даннера [9].

Энтальпия и энтропия азота рассчитаны в однофазной области и вдоль линий кипения и конденсации.

В табл. 1 приведены расчетные значения плотности, энтальпии и энтропии для различных значений давлений и температур. При сравнении с экспериментальными данными [10] к расчетным значениям $H$ добавлена теплота сублимации азота $h_{0}^{0}=247,6$ кДж/кг при $T=0 \mathrm{~K}$, поскольку для идеально-газовой энтальпии, используемой в [10], принята ненулевая константа отсчета.

Отклонения расчетных значений $H$ и $S$ вдоль линий кипения и конденсации от эксперимен-

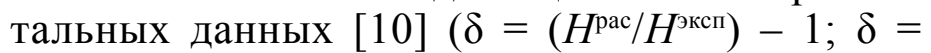

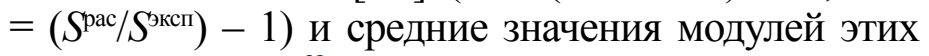
отклонений $\bar{\delta}=\sum_{n=1}^{N_{\delta}}\left|\delta\left(T_{n}\right)\right| / N_{\delta}$ показывают, что приведенные результаты расчетов удовлетворительно согласуются с опытными данными.

\section{Выводы}

Анализ полученных результатов показывает возможность успешного применения строгих статистико-механических методов к описанию свойств молекулярных систем в различных фазовых состояниях. При использовании предложенного метода вполне достаточно минимального набора исходных данных (давление, температура). Рассчитанные термодинамические характеристики жидкого и газообразного азота могут быть использованы для разработки перспективных видов экологически чистых и пожаробезопасных транспортных средств с криогенными силовыми установками. 
Табл. 1. Сравнение расчетных и экспериментальных значений плотности, энтальпии и энтропии

\begin{tabular}{|c|c|c|c|c|c|c|}
\hline$T, \mathrm{~K}$ & $D, \kappa \Gamma / \mathrm{M}^{3}$ & $D[10], \kappa \Gamma / \mathrm{M}^{3}$ & $H$, кДж/кг & $H[10]$, кДж/кг & $S$, кДж/(кг·К $)$ & $S[10]$, кДж/(кг K) \\
\hline \multicolumn{7}{|c|}{$p=0,1 \mathrm{MПа}$} \\
\hline 70 & 838,45 & 837,86 & 126,36 & 110,5 & 2,757 & 2,628 \\
\hline 80 & 4,353 & 4,37 & 328,76 & 328,3 & 5,463 & 5,457 \\
\hline 90 & 3,833 & 3,85 & 339,56 & 338,8 & 5,59 & 5,58 \\
\hline 100 & 3,429 & 3,44 & 350,19 & 349,5 & 5,703 & 5,693 \\
\hline 110 & 3,104 & 3,11 & 360,76 & 360,2 & 5,803 & 5,795 \\
\hline 120 & 2,837 & 2,84 & 371,28 & 370,8 & 5.895 & 5,887 \\
\hline 200 & 1,6882 & 1,69 & 454,87 & 454,5 & 6,429 & 6,422 \\
\hline 250 & 1.34885 & 1,35 & 506,84 & 506,7 & 6,661 & 6,655 \\
\hline 300 & 1,1234 & 1,12 & 558,77 & 558,8 & 6,85 & 6,844 \\
\hline 350 & 0,9626 & 0,96 & 610,77 & 610,9 & 7,011 & 7,005 \\
\hline \multicolumn{7}{|c|}{$p=5 \mathrm{M \Pi а}$} \\
\hline 70 & 849,657 & 847,64 & 130,28 & 114,4 & 2,73 & 2,60 \\
\hline 80 & 807,857 & 806,29 & 148,1 & 134,8 & 2,968 & 2,873 \\
\hline 90 & 761,098 & 760,87 & 167,86 & 155,1 & 3,2 & 3,112 \\
\hline 100 & 710,637 & 711,62 & 188,69 & 175,7 & 3,42 & 3,329 \\
\hline 110 & 655,410 & 654,93 & 210,28 & 197,6 & 3,625 & 3,538 \\
\hline 120 & 591,739 & 582,07 & 233,14 & 222,6 & 3,824 & 3,755 \\
\hline 200 & 93,677 & 93,42 & 432,42 & 429,8 & 5,184 & 5,168 \\
\hline 250 & 69,877 & 69,58 & 492,15 & 491,1 & 5,451 & 5,442 \\
\hline 300 & 56,587 & 56,35 & 548,53 & 548,2 & 5,657 & 5,65 \\
\hline 350 & 47,8325 & 47,67 & 603,47 & 603,6 & 5,827 & 5,821 \\
\hline \multicolumn{7}{|c|}{$p=10 \mathrm{MПа}$} \\
\hline 70 & 860,083 & 856,49 & 134,36 & 118,5 & 2,705 & 2,575 \\
\hline 80 & 820,748 & 818,06 & 154,63 & 138,4 & 2,935 & 2,841 \\
\hline 90 & 777,58 & 776,24 & 170,84 & 158,2 & 3,161 & 3,074 \\
\hline 100 & 732,34 & 732,53 & 190,9 & 178,0 & 3,372 & 3,282 \\
\hline 110 & 685,119 & 685,75 & 211,25 & 198,3 & 3,566 & 3,476 \\
\hline 120 & 635,141 & 633,03 & 231,8 & 219,8 & 3,745 & 3,663 \\
\hline 200 & 207,29 & 199,54 & 408,64 & 405,8 & 4,886 & 4,869 \\
\hline 250 & 142,62 & 140,59 & 478,13 & 477,1 & 5,198 & 5,188 \\
\hline 300 & 112,91 & 111,69 & 539,13 & 539,1 & 5,421 & 5,414 \\
\hline 350 & 94,48 & 93,7 & 596,92 & 597,6 & 5,599 & 5,596 \\
\hline
\end{tabular}




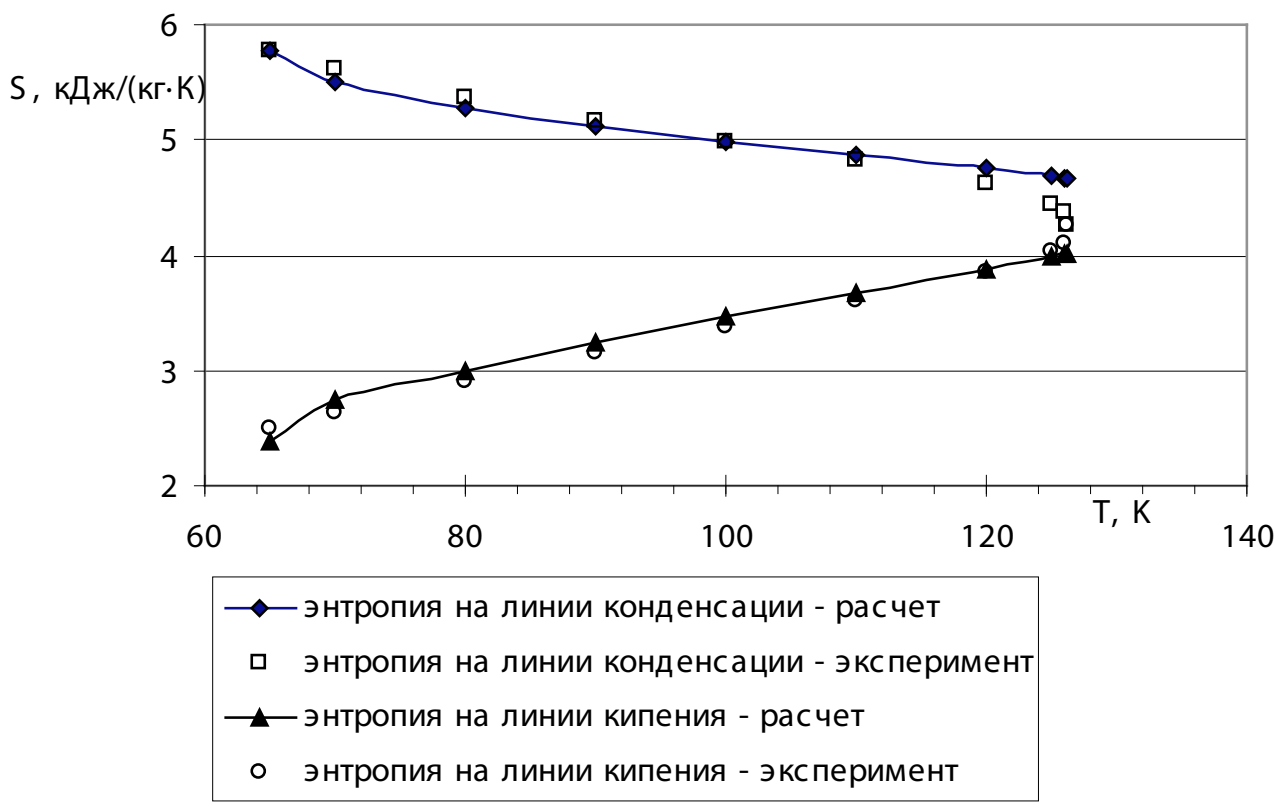

Рис. 1. Энтропия азота на линии кипения и конденсации.

Экспериментальные данные [10].

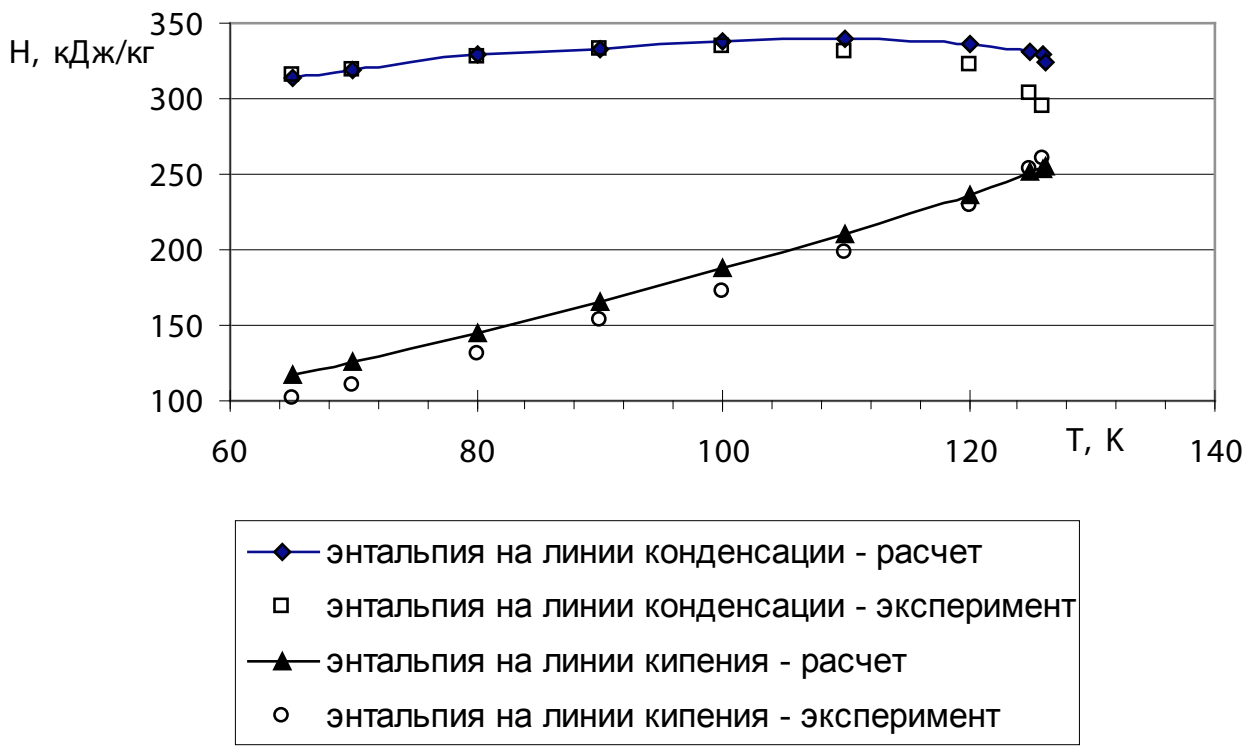

\section{Рис. 2. Энтальпия азота на линии кипения и конденсации. Экспериментальные данные [10].}

Табл. 2. Средние значения модулей отклонений $\bar{\delta}$ энтальпии и энтропии азота

\begin{tabular}{|c|c|c|c|c|}
\hline \multirow{2}{*}{} & \multicolumn{2}{|c|}{ Линия кипения } & \multicolumn{2}{c|}{ Линия конденсации } \\
\cline { 2 - 5 } & $\mathrm{H}$ & $\mathrm{S}$ & $\mathrm{H}$ & $\mathrm{S}$ \\
\hline $\bar{\delta}$ & 0,078 & 0,028 & 0,031 & 0,028 \\
\hline
\end{tabular}




\section{ЛИТЕРАТУРА}

1. Plummer M.C. Cryogenic heat engine experiment / M.C. Plummer, C.P. Koehler, D.R. Flanders // Proc. of 1997 Cryogenic Eng. Conf., Portland, July 1997, USA. - P. 7.

2. Williams J. Frost-Free Cryogenic Heat Exchanger for Automotive Propulsion / J. Williams, C. Knovlen, A.T. Mattick, A. Hertzberg // Proc. of 33rd AIAA/ASEE Joint Propulsion Conf. @ Exhibit, July 6-9, 1997, Seattle, USA. - P. 18.

3. Туренко A.Н. Экологически чистый криогенный транспорт: современное состояние проблемы /А.Н. Туренко, А.И. Пятак, И.Н. Кудрявцев // Вестн. Харьк. автомоб.-дорожн. техн. ун-та. 2000. - Вып. 12-13. - С. 42-47.

4. Сухов А.П. Выхлоп чище воздуха // За рулем. - 2001.- № 2. - С. 40-42.

5. Бондаренко С.И. Криоавтомобиль: будем ездить на азоте? / С.И. Бондаренко, И.Н. Кудрявцев // Автоцентр. - 2000.- № 40. - С. 32-33.

6. Бондаренко С.И. Новый вид пожаробезопасного и экологически чистого транспортного средства для аэропортов / С.И. Бондаренко, И.Н. Кудрявцев, А.И. Пятак, И.И. Тимченко, А.П. Кудряш // Пробл. машиностроения. - 2002. - Т. 5. - № 7. - С. 92-95.

7. Маринин B.C. Теплофизика альтернативных энергоносителей. - Харьков: Форт. - 1999. - 212 с.

8. Левтеров A.M. Расчетная оценка теплофизических свойств азота, как рабочего тела поршневого криодвигателя. Часть I. Математическая модель фазовых равновесий / А.М. Левтеров, К.Р. Умеренкова // Пром. теплотехника. - 2013. T. 35, № 4. - С. 90 - 95 .

9. Левтеров А.M. Расчетная оценка теплофизических свойств азота, как рабочего тела поршневого криодвигателя. Часть II. Вычисление теплоемкости / А.М. Левтеров, К.Р. Умеренкова // Пром. теплотехника. - 2014. - Т. 36, № 2. - С. 93 -100 .

10. Термодинамические свойства азота / В.В. Сычев, А.А. Вассерман, А.Д. Козлов. - М.: Издат. стандартов. - 1977. - 720 с.

\section{CALCULATION ASSESSMENT OF THERMAL PROPERTIES OF NITROGEN AS THE BODY WORK PISTON CRYOGENIC ENGINE. PART III. THE CALCULATION OF ENTHALPY AND ENTROPY}

\section{A. Levterov, K. Umerenkova}

The A. N. Podgorny Institute for Mechanical Engineering Problems of the National Academy of Sciences of Ukraine, Kharkiv, Ukraine, ul. Pozharsky, 2/10, Kharkov, 61046, Ukraine

In article theoretical model for description of the thermodynamic properties of liquid and gaseous nitrogen which used as a working body for transport piston power plants is proposed. Mathematical model for description of heat capacity is based on the unified statistical mechanical approach within the thermodynamic perturbation theory without invoking of any empirical parameters. The numerical calculations which accomplished for enthalpy and entropy of the molecular nitrogen agree with the available experimental data. Elaborated calculation procedures are used at creation of new kind of ecological and fireproof vehicle.

References 10, tables 2, figure 2 .

Key words: reciprocating cryogenic engine, nitrogen, thermal characteristics, working body, enthalpy, entropy, mathematical model.

1. Plummer M.C. Cryogenic heat engine experiment / M.C. Plummer, C.P. Koehler, D.R. Flanders 
// Proc. of 1997 Cryogenic Eng. Conf., Portland, July 1997, USA. - P. 7.

2. Williams $J$. Frost-Free Cryogenic Heat Exchanger for Automotive Propulsion / J. Williams, C. Knovlen, A.T. Mattick, A. Hertzberg // Proc. of 33rd AIAA/ASEE Joint Propulsion Conf. @ Exhibit, July 6-9, 1997, Seattle, USA. - P.18.

3. Turenko A.N. Eco-friendly cryogenic transport: the current state of the problem / A.N. Turenko, A.I. Pjatak, I.N. Kudryavtsev // Vestnik Kharkov Avtomobilno-doroznogo tekhnicheskogo universiteta - 2000. - Issue. 12-13. - P. 42-47. (Rus.).

4. Suhov A.P. Vyhlop is cleaner than air // Za rulom. - 2001. - № 2. - P. 40-42. (Rus.).

5. Bondarenko S.I. Krioavtomobil: we shall go by nitrogen? / S.I. Bondarenko, I.N. Kudryavtsev // Avto centr. - 2000. - № 40. - P. 32-33. (Rus.).

6. Bondarenko S.I. New a kind of a fireproof and non-polluting vehicle for the airports / S.I. Bondarenko, I.N. Kudryavtsev, A.I. Pjatak, I.I. Timchenko,
A.P. Kudrjash // Problem mashinostroeniya. - 2002. - V. 5. - № 7. - P. 92-95. (Rus.).

7. Marinin V.S. Thermal physics of alternative energy sources. - Kharkov: Fort. $-1999 .-212$ p. (Rus.).

8. Levterov A.M. Calculation assessment of thermal properties of nitrogen as the body work piston cryogenic engine. Part I. Mathematical model of phase equilibrium / A.M. Levterov, K.R. Umerenkova // Promyshlennaya teplotekhnika. 2013. -V. 35, № 4. - P. 90-95. (Rus.).

9. Levterov A.M. Astimation of thermophysical properties of nitrogen as the working body for piston cryo-engine. Part II. The calculation of heat capacity / A.M. Levterov, K.R. Umerenkova // Prom. teplotekhnika. - 2014. -V. 36, № 2. - P. 93-100. (Rus.).

10. Thermodynamic Properties of Nitrogen / V.V. Sychev, A.A. Wasserman, A.D. Kozlov etc. - M: Izdatelstvo Standartov. - 1977. - 350 p. (Rus.) 